\title{
Paradoxical effects of base rates and representation in category learning
}

\author{
MARK K. Johansen \\ Cardiff University, Cardiff, Wales \\ AND \\ Nathalie Fouquet and David R. Shanks \\ University College London, London, England
}

\begin{abstract}
The inverse base rate effect (IBRE; Medin \& Edelson, 1988) continues to be a puzzling case of decision making on the basis of conflicting information in human category learning. After being trained via feedback over trials to assign combinations of cues to high- and low-frequency categories, participants tend to respond with the low-frequency category to an otherwise perfectly conflicting pair of test cues, contrary to the category base rates. Our Experiment 1 demonstrated that decision making on the basis of an explicit summary of the cue-outcome and outcome base rate information from the standard learning task does not result in the effect. The remaining experimental conditions evaluated the necessary and sufficient conditions for the effect by systematically exploring experimental deviations between the standard learning task and the pure decision-making task. In partial disagreement with both recent theoretical accounts of the effect (Juslin, Wennerholm, \& Winman, 2001; Kruschke, 1996), these experiments indicate that asymmetric outcome representation and profound base rate neglect are individually necessary and jointly sufficient conditions. Broader theoretical implications are discussed.
\end{abstract}

Categorizing ambiguous stimuli is a common form of decision making, particularly because real-world categories are complex and differ widely in their base rates of occurrence. Although generally adaptive, human decision making is sometimes surprising and paradoxical, especially when faced with conflicting information. The long research history for heuristics and biases (e.g., Kahneman \& Tversky, 1973) suggests that they facilitate behavior with limited computational capacity even in complicated environments. So, not surprisingly, cases of puzzling decision making occur in response to conflicting information in category learning. The inverse base rate effect is one of those cases.

Building on Binder and Estes (1966), Medin and Edelson (1988) reported several category-learning experiments that resulted in an inverse base rate effect (IBRE) - that is, data contrary to the use of prior outcome frequencies in response to a conflicting pair of cues both of which were previously perfect outcome predictors. This effect has since been replicated many times (Bohil, Markman, \& Maddox, 2005; Juslin, Wennerholm, \& Winman, 2001; Kalish, 2001; Kruschke, 1996; Medin \& Bettger, 1991; Nelson, 1993; Shanks, 1992) and continues to spark new theories and research (Bohil et al., 2005; Kruschke, 2001, 2003; Winman, Wennerholm, \& Juslin, 2003; Winman, Wennerholm, Juslin, \& Shanks, 2005).

The IBRE has typically been found in a disease diagnosis paradigm based on the abstract category structure shown at the top of Figure 1 (terminology from Kruschke, 1996). On each trial in a training phase, participants see a pair of symptoms (cues) for a patient, diagnose the patient's disease (outcome), and then receive corrective feedback. Over trials, the participants learn to diagnose multiple cases of (C)ommon and (R)are diseases with different outcome base rates (frequencies) of, typically, 3 to 1 . A given pair of symptoms always and only occurs with the given disease. So in Figure 1, "PC" is said to be the (P)erfectly predictive cue of the (C)ommon outcome and "PR" the corresponding cue for the (R)are outcome. The second cue in the pair of symptoms is represented by "I" and is (I)mperfectly predictive of the outcome because it also occurs with another outcome that has a different base rate.

Following the training phase, the participants are tested on new combinations of the training symptoms without feedback. Most important is PC+PR (Figure 1, top) which pits the $(\mathrm{P})$ erfectly predictive cue of the $(\mathrm{C})$ ommon outcome against the (P)erfectly predictive cue of the (R)are outcome. Since both PC and PR have been perfectly correlated with $\mathrm{C}$ and $\mathrm{R}$, respectively, during training and have been equally predictive, only the difference in outcome base rates differentiates them and plausibly invites (C)ommon responding because of its higher base rate. However, participants tend to respond to the conflicting PC+PR with (R)are-hence, the inverse in IBRE.

M. K. Johansen, johansenm@cardiff.ac.uk 

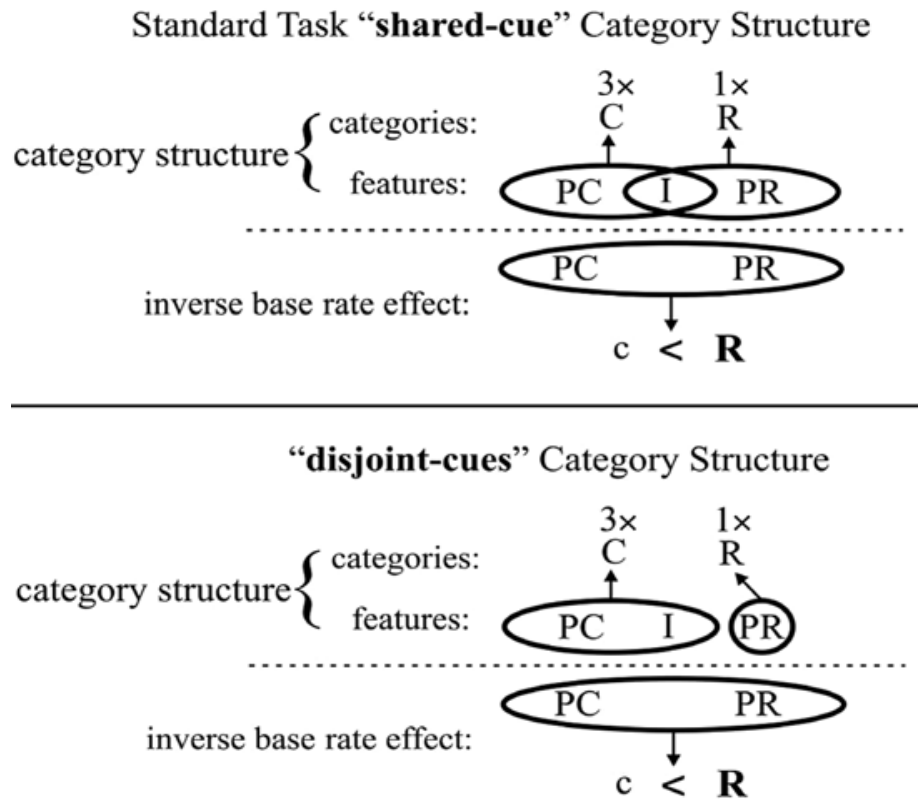

\begin{abstract}
Figure 1.Top:Abstract category structure, designated the "shared-cue" category structure, plus result for the standard inverse base-rate effect. Bottom: "Disjoint-cues" category structure matching the hypothesized asymmetric representation formed in the standard task. The following terminology is from Kruschke (1996): " $C$ " represents a (C)ommon outcome $(3 \times)$ and " $R$ " a $(R)$ are outcome $(1 \times)$. "PC" refers to the $(P)$ erfectly predictive cue of the (C)ommon outcome, "PR," the (P)erfectly predictive cue of the (R)are outcome, and "I" the (I)mperfectly predictive, shared cue. Cues within an ellipse occur together. This figure was inspired by Kruschke's (2001) Figure 1.
\end{abstract}

The typical results for the remaining standard testing trials are less surprising. For an (I)mperfectly predictive cue by itself (I), participants tend to respond (C)ommon. Likewise, for the combination of all three cues, I+PC $+\mathrm{PR}$, participants tend to respond $(\mathrm{C})$ ommon. We will refer to this as the standard IBRE task and results, corresponding to what we call the shared-cue category structure (Figure 1, top).

A clear indication of the theoretical difficulty and importance of the IBRE can be had from the fact that there have been as many attempts to model it (Anderson, 1990; Gluck, 1992; Juslin et al., 2001; Kalish, 2001; Kruschke, 1996, 2001, 2003; Markman, 1989; Medin \& Edelson, 1988; Myers, Lohmeier, \& Well, 1994; Shanks, 1992; Winman et al., 2003) as to empirically clarify it (Bohil et al., 2005; Juslin et al., 2001; Kalish, 2001; Kruschke, 1996, 2001; Medin \& Bettger, 1991; Medin \& Edelson, 1988; Nelson, 1993; Shanks, 1992; Winman et al., 2005). This is particularly telling since the difficulty of accounting for the IBRE essentially reduces to handling a single problematic data point $(\mathrm{PC}+\mathrm{PR})$ without creating an entire theoretical structure that has no application beyond that data point. Because it is so problematic, we will argue that the effect goes to the heart of several important issues in human learning and decision making.

We have assumed the paradigm that category learning corresponds to the formation of a category representation, followed by decision making based on that representation.
From this perspective, the IBRE is the result of the representation formed during learning or decision making on that representation at test (or both). Essentially, all previous attempts to explain the effect have emphasized either representation or decision making but have not sought to compare them directly, which is our goal here.

Before considering these low-level representation and high-level decision-making perspectives in detail, it is important to emphasize that both are still conceptually viable, even though evidence has previously been presented against specific formal instantiations for both (e.g., Juslin et al., 2001; Kruschke, 1996, 2001, 2003; Winman et al., $2003,2005)$. In addition, we will present further data that are conceptually incompatible with both (Juslin et al., 2001; Kruschke, 1996).

\section{Representation-Based Explanations}

The majority of attempts to explain the IBRE have assumed an asymmetry in the representation formed for the $(\mathrm{C})$ ommon and $(\mathrm{R})$ are outcomes during training. For example, in the hypothesized asymmetric representation shown at the bottom of Figure 1 (inspired in part by Kruschke, 2001, Figure 1), the (I)mperfectly predictive cue is only in the representation for (C)ommon and not in that for (R)are, even though it occurred with (R)are during training. In other words, $\mathrm{C}$ is represented by both I and PC, but R only by PR. This asymmetry makes the conflicting 
test $\mathrm{PC}+\mathrm{PR}$ more similar to the representation for $(\mathrm{R})$ are than to that for $(\mathrm{C})$ ommon, so $\mathrm{R}$ responses predominate.

Medin and Edelson (1988) accounted for the IBRE with a kind of asymmetric representation in an extension of the context model (Medin \& Schaffer, 1978), which assumes exemplar representation - that is, storage of category instances in proportion to their base rates. Although this mechanism is not helpful in explaining the effect, exemplar models usually assume selective attention to stimulus features on the basis of their outcome predictiveness during training (see the generalized context model [Nosofsky, 1984, 1986] and ALCOVE [Kruschke, 1992]). More predictive cues receive more attention and influence similarity more strongly. Medin and Edelson formalized the similarity parameters in the context model so that the (I)mperfectly predictive cue receives more attention in the context of PC than in the context of PR. Because of this, I is more strongly associated with (C)ommon than (R)are, and I's absence makes PC+PR less similar to the representation for $\mathrm{C}$ than to that for $\mathrm{R}$.

Medin and Edelson's (1988) idea has persisted in many more recent accounts of the IBRE as differential similarity based on selective attention, resulting in asymmetric outcome representations. For example, Anderson's (1990) well-known rational model can account for the effect by allowing different similarity gradients around the representations for (C)ommon and (R)are. Furthermore, Shanks's (1992) attentional connectionist model can account for the effect as a result of a stronger associative weight between $\mathrm{PR}$ and $\mathrm{R}$ than between PC and $\mathrm{C}$ as a consequence of differences in expectation from selective attention to the cues. Most important, Kruschke's (1996) ADIT model, arguably the best quantitative account of the effect, has been strongly influenced by this perspective.

The ADIT model (Kruschke, 1996) extended Gluck and Bower's (1988) component cue model of associative learning by adding mechanisms for error-driven selective attention, on top of error-driven association learning, and systematic outcome base rate influences on responding. It explains the IBRE using two major principles: (1) asymmetric representation, where the $(\mathrm{C})$ ommon outcome is represented by its typical features, both PC and I, whereas the (R)are outcome is represented predominantly by the single feature distinguishing it from the (C)ommon, PR; (2) systematic base rate influences on decision making, moderated only by the number of cues in the stimulus. The evidence for these principles was further bolstered by ADIT's ability to also account for the apparent base rate neglect found in a partial replication of Experiment 1 from Gluck and Bower - in particular, invoking not base rate neglect but, rather, asymmetric representation and systematic base rate influences.

Broadly, the hypothesized asymmetric representation in ADIT (see Figure 1, bottom) results from error-driven selective attention that moderates the error-driven learning of cue-outcome associative weights. The asymmetric representation occurs during training because the (C)ommon outcome tends to be learned before the (R)are as a result of C's higher base rate and C tends to be encoded in terms of both of its cues, PC and I. Since I has already been associated with (C)ommon, I's occurrence on (R)are trials generates error (to the degree that it predicts C). ADIT uses this error to shift attention away from the source of the error, I, toward PR, hence moderating the activation of these cues before updating the cue-outcome associations. So the burden of predicting $\mathrm{R}$ is carried mostly by $\mathrm{PR}$, and the associative weight from I to $\mathrm{R}$ is much weaker than that from I to C - hence, the asymmetry in representation.

In addition to the indirect/implicit influence of the outcome base rates on the formation of the asymmetric representation, Kruschke (1996) also hypothesized that participants explicitly know and apply base rate knowledge during the testing phase; that is, they know that (C)ommon > (R)are, with this being inversely moderated by the number of cues in the stimulus. However, for the critical PC + PR test, the base-rate-driven tendency to respond (C)ommon is overridden by the asymmetric representation's tendency to produce $(\mathrm{R})$ are responses.

\section{Decision-Making-Based Explanations}

Binder and Estes (1966) hypothesized that responding (R)are to the conflicting cues PC+PR is the result of a high-level-reasoning novelty effect: Because the conflicting PC + PR case is a new combination of cues - so, in some senses, rare - (R)are responses predominate. But Medin and Edelson (1988) added the new testing case consisting of all three cues, I+PC $+\mathrm{PR}$, and found dominantly (C)ommon responding, inconsistent with a simple novelty effect. However, Juslin and colleagues (Juslin et al., 2001; Winman et al., 2003,2005 ) have pursued a high-level-reasoning approach involving a more sophisticated kind of novelty effect.

Juslin et al. (2001) proposed an eliminative inference process to explain the IBRE, formalized in the ELMO model. By this account, participants form high-level inference rules during the learning phase: $\mathrm{I}+\mathrm{PC} \rightarrow(\mathrm{C})$ ommon, and $\mathrm{I}+\mathrm{PR} \rightarrow(\mathrm{R})$ are. The outcome base rates only indirectly influence responding: These inference rules are part of an active set in working memory, with probabilities proportional to the base rates of their respective outcomes during training. Since (C)ommon has a higher base rate than (R)are, the active set is more likely to contain $\mathrm{C}$ inference rules than $\mathrm{R}$ ones when participants are confronted with the critical test case PC $+\mathrm{PR}$. By an eliminative inference which is essentially a novelty effect, $\mathrm{PC}+\mathrm{PR}$ is judged to be sufficiently dissimilar to the inference rule $\mathrm{I}+\mathrm{PC} \rightarrow \mathrm{C}$ to eliminate the (C)ommon response. The remaining possible responses are then chosen among at random. Hence, there are more (R)are responses than (C)ommon because the inference rule $\mathrm{I}+\mathrm{PR} \rightarrow \mathrm{R}$ is less likely to be in the active set and less likely to be eliminated as a possible response.

The key differences between the theoretical positions embodied in ADIT (Kruschke, 1996) and ELMO (Juslin et al., 2001) are (1) representation, whereby ELMO assumes that inference rules are always composed of pairs of cues, so that the representation for a given outcome, if active at all, is based on two cues symmetrically for either $\mathrm{I}+\mathrm{PC} \rightarrow \mathrm{C}$ or $\mathrm{I}+\mathrm{PR} \rightarrow \mathrm{R}$, whereas ADIT assumes asymmetric representation in that $\mathrm{C}$ is represented mostly in terms of two cues (PC+I) but R by one (PR), and (2) decision making, whereby ELMO assumes that high-level reasoning - that is, eliminative inference - compensates 
at test for low similarity to the (C)ommon inference rule and no similarity to the nonactive (R)are rule, whereas ADIT assumes low-level similarity assessment at test to the asymmetric $(\mathrm{C})$ ommon and $(\mathrm{R})$ are representations formed during learning. Plus, ADIT assumes systematic base rate influences on decision making at test, whereas ELMO assumes base rate neglect at test.

Kruschke (2001) demonstrated that eliminative inference, as formalized in ELMO (Juslin et al., 2001), predicts an IBRE even when there is no (I)mperfect/shared symptom during training, whereas data from a new experiment were not consistent with this prediction. Experiment 2 below also provides converging evidence for this result.

Winman et al. (2003) agreed with this conclusion for eliminative inference, as formalized in ELMO, but still critiqued several of the principles in Kruschke's (1996) ADIT model. More important, Winman et al. (2005) provided further evidence of a high-level-reasoning component in the IBRE: They showed that the between-subjects tendency to exhibit or not exhibit the IBRE is correlated with a tendency to form rules in a separate task differentiating rule-based from similarity-based processing (the Shanks \& Darby [1998] task).

\section{Research Purpose}

Prior research on the IBRE has suggested that further evaluation of explanations based on low-level representation and high-level decision making is needed. Hence, the purpose of our research was to explore the necessary and sufficient conditions for the IBRE, particularly in terms of the representation for the task (symmetric or asymmetric representations of the high- and low-base-rate outcomes) and the decision making on the representation (particularly outcome base rate use or neglect).

Our general methodology was to evaluate systematic experimental deviations from the standard IBRE learning task. As a preview, Experiment 1 evaluated decision making in the IBRE by having participants respond to the test cases using an explicit cue-outcome and base rate summary. Experiment 2 evaluated representation by actually making the cue-outcome relations match the hypothesized representation asymmetry (Figure 1, bottom), and Experiment 3 manipulated both representation symmetry and base rate use.

\section{EXPERIMENT 1 Evaluating Decision Making}

In this experiment, participants responded to the standard test cases on the basis of the simplest possible summary of the cue-outcome and base rate information from the standard IBRE task (Figure 1, top), rather than learning that information over a series of trials with corrective feedback. This summary described the disease diagnosis paradigm and conveyed essentially three pieces of information, where the symbols in brackets indicate the mapping onto the abstract category structure (Figure 1, top): Patients with the symptoms dizziness [I] and sore muscles $[\mathrm{PC}]$ have the disease terrigitis $[\mathrm{C}]$; patients with the symptoms dizziness [I] and stuffy nose [PR] have the disease coralgia $[R]$; and three times as many patients have terrigitis [C] as have coralgia [R]. The participants in the pure decision-making condition responded to the test cases with the summary available. In the memorization condition, the participants responded to the test cases on the basis of their memory of a summary of a different, nonmedical cover story that had the same abstract cues and outcomes as those in the pure decision-making condition.

\section{Method}

Participants. There were 33 (unpaid) and 30 (£1) participants in the pure decision-making and memorization conditions, respectively - mainly, psychology students at University College London (UCL).

Materials and Procedure. The pure decision-making condition had two versions of the single-page instructions and test cases, which differed only in the symptom and disease names, the order of the information for the common and rare diseases in the first paragraph, and the order of the possible answers on the testing trials. The summary (see Appendix A) contained information corresponding to the abstract relationships $\mathrm{I}+\mathrm{PC} \rightarrow \mathrm{C}$ and $\mathrm{I}+\mathrm{PR} \rightarrow \mathrm{R}$ and the base rates (C:R, 3:1; see Figure 1, top) and was followed by a series of seven testing trials in the order: $\mathrm{PC}+\mathrm{PR}, \mathrm{PC}+\mathrm{I}+\mathrm{PR}, \mathrm{I}, \mathrm{I}+\mathrm{PR}$, $\mathrm{PC}, \mathrm{PR}$, and $\mathrm{I}+\mathrm{PC}$. The memorization condition used a different cover story (an ethnologist studying tribal face painting) but had the same abstract cue-outcome relations. The participants in the memorization condition were given $2 \mathrm{~min}$ to memorize the summary before the test, whereas the summary was constantly available in the decision-making condition.

\section{Results and Discussion}

The testing trial response proportions from both conditions are shown in Table 1, together with the results of Kruschke's (1996, Experiment 1) standard replication of the IBRE. Unlike the standard task, which results in more (R)are than (C)ommon responses to $\mathrm{PC}+\mathrm{PR}(.35 \mathrm{C}$ response proportion; Kruschke, 1996), the pure decisionmaking task resulted in significantly more (C)ommon than (R)are responses, consistent with the outcome base rates of $\mathrm{C}>\mathrm{R}\left[.94 \mathrm{C}, H_{0}=0.5 ; \chi^{2}(1)=25.49, p<.0001\right]$, and the memorization task resulted in marginally more $\mathrm{C}$ than $\mathrm{R}$ responses $\left[.67 \mathrm{C}, H_{0}=0.5 ; \chi^{2}(1)=3.33, p=\right.$

Table 1

Experiment 1: Testing Trial Response Proportions

\begin{tabular}{llllcr}
\hline & \multicolumn{4}{c}{ Condition } \\
\cline { 2 - 3 } \cline { 5 - 6 } Cues/Outcomes & \multicolumn{2}{c}{ Decision Making } & & Memorization \\
\cline { 2 - 3 } \cline { 5 - 6 } PC+I & (C)ommon & (R)are & & (C)ommon & (R)are \\
PR+I & $.97(.98)$ & $.03(-)$ & & 1.00 & .00 \\
I & $.06(-)$ & $.94(.95)$ & & .03 & .97 \\
PC & $.91(.75)$ & $.09(.17)$ & & .80 & .20 \\
PR & $.97(.93)$ & $.03(.03)$ & & 1.00 & .00 \\
PC+PR & $.06(.04)$ & $.94(.91)$ & & .03 & .97 \\
I+PC+PR & $.94(.35)$ & $.06(.61)$ & & .67 & .33 \\
\hline
\end{tabular}

Note- "PC" is the $(\mathrm{P})$ erfectly predictive cue of the $(\mathrm{C})$ ommon outcome, "PR" is the $(\mathrm{P})$ erfectly predictive cue of the $(\mathrm{R})$ are outcome, and "I" is the (I)mperfectly predictive cue of both outcomes. The numbers in parentheses are from Kruschke (1996, Experiment 1), a standard replication of the inverse base rate effect. Also note that Kruschke's (1996) response proportions for a given trial do not quite sum to one, because a small number of other answers were possible and occurred. 
.068], although significantly more than Kruschke's (1996) Experiment 1 response proportions, which will be used here as a standard reference throughout $[.67 \mathrm{C}>.35 \mathrm{C}$; $\left.\chi^{2}(1)=7.73, p=.0054\right]$. Finally, the memorization condition resulted in significantly less base-rate-consistent responding than did the decision-making condition [.67 $\left.\mathrm{C}<.94 \mathrm{C} ; \chi^{2}(1)=7.58, p=.0059\right]$.

The decision-making condition resulted in marginally more (C)ommon responses to the (I)mperfectly predictive cue by itself than did the standard learning task [.91 C > $\left..75 \mathrm{C} ; \chi^{2}(1)=3.40, p=.0652\right]$, as did the combination of all three cues, I+PC+PR $\left[.91 \mathrm{C}>.58 \mathrm{C} ; \chi^{2}(1)=11.20\right.$, $p<.0008]$. Overall, the memorization condition resulted in less but still base-rate-consistent responding (Table 1).

Neither the decision-making nor the memorization tasks resulted in an IBRE but, rather, in base-rate-consistent responding, presumably because the base rate information was explicitly summarized and, hence, used. However, comparing the two conditions suggests that memorization reduced the incorporation of the base rate information into the decision-making process and that the absence of the IBRE in the pure decision-making condition was not simply the result of a failure to adequately encode the summary information in working memory.

So decision making on summary information corresponding to explicit symmetric representation and explicit base rate information was not sufficient to produce the IBRE. Clearly, some other aspects of the standard learning task are necessary conditions, and the effect is not simply another case of nonnormative decision making. Accounts of the IBRE emphasizing learning have all hypothesized some form of asymmetric representation, and this was evaluated in Experiment 2. The remaining experimental conditions were all systematic deviations between the pure decision-making task, which does not result in the effect, and the standard learning task, which does.

\section{EXPERIMENT 2 Evaluating the Representation Component}

Since asymmetric representation has been a dominant explanation of the IBRE, the main purpose of Experiment 2 was to directly evaluate this by changing the category structure to match explicitly the asymmetric representation (Figure 1, bottom) hypothesized to be formed in the standard task (most specifically by Kruschke, 1996, 2001). We will refer to the bottom of Figure 1 as the disjoint-cues category structure, in contrast to the standard, shared-cue structure at the top. Disjoint-cues tasks simply have two $(\mathrm{P})$ erfectly predictive cues associated with the (C)ommon outcome but only a single (P)erfectly predictive cue associated with (R)are and no (I)mperfectly predictive/shared cues. If asymmetric representation is a sufficient and necessary condition for the IBRE, tasks that explicitly formalize it by using the disjoint-cues category structure should result in a particularly robust effect.

There were four conditions in this experiment, designed to evaluate this category structure under a wide range of settings: the pure decision-making and summary memorization conditions were essentially identical to those in Ex- periment 1, except for the change to the disjoint-cues category structure. Likewise, the feedback-learning condition was essentially identical to the standard learning task (e.g., Kruschke, 1996), except for the change to the disjoint-cues category structure, and the observational-learning condition was essentially identical to the feedback-learning condition, except that outcome anticipation and feedback were eliminated.

\section{Method}

Participants. The decision-making, memorization, feedback, and observational learning conditions had, respectively, 33 (unpaid), $30(£ 1), 16(£ 3)$, and $16(£ 3)$ participants, ${ }^{1}$ mainly psychology students.

Materials and Procedure. The decision-making and memorization conditions were identical to those in Experiment 1, except that the summary information was changed to reflect the asymmetric representation of the $(\mathrm{C})$ ommon and $(\mathrm{R})$ are outcomes as embodied in the disjoint-cues category structure (Figure 1, bottom): The (R)are outcome was stated to have only a single cue associated with it (e.g., stuffy nose $\rightarrow$ coralgia), whereas the (C)ommon outcome was still associated with two cues (e.g., sore muscles and dizziness $\rightarrow$ terrigitis). The testing trials are listed in the left column of Table 2.

The materials and procedure for the feedback-learning condition were very similar to those in Kruschke's (1996) standard IBRE learning task. The main difference was that the abstract category structure was changed to the disjoint-cues category structure in Table 3. Each row in Table 3 specifies an instance composed of cues that were either present, coded as $1 \mathrm{~s}$, or absent, coded as $0 \mathrm{~s}$. Note that some of the instances were identical, so some cases had relatively high base rates, labeled (C)ommon, whereas other cases had relatively low base rates, labeled (R)are. There were no cues associated with more than one outcome; that is, there were no (I)mperfectly predictive, shared cues. All the cues were perfectly predictive of their respective outcomes, $\mathrm{PC}$ for $(\mathrm{P})$ erfectly predictive cue of a (C)ommon outcome and $\mathrm{PR}$ for $(\mathrm{P})$ erfectly predictive cue of a $(\mathrm{R})$ are outcome. Each cue and outcome are subscripted with 0.5 if the cue was paired with another cue during training and subscripted with 1 if the cue occurred by itself.

The six abstract cues and four outcomes in Table 3 were randomly assigned to symptoms and diseases, respectively, from the following sets for each participant: symptoms (skin rash, fever, back pain, blurred vision, insomnia, ear aches, sore muscles, sneezing, wheezing, twitching, stuffy nose, and dizziness) and diseases (F, G, H, and $\mathrm{J}$ ).

Stimuli were presented on a computer screen, with symptoms arranged in a column and in random order from trial to trial. Below the column of cues, there was the response prompt "Diagnose as one of

Table 2

Experiment 2: Testing Trial Response Proportions for Summary Based Conditions

\begin{tabular}{lccccc}
\hline & \multicolumn{4}{c}{ Condition } \\
\cline { 2 - 3 } \cline { 5 - 6 } Cues/Outcomes & \multicolumn{2}{c}{ Decision Making } & & \multicolumn{2}{c}{ Memorization } \\
\cline { 2 - 3 } \cline { 5 - 6 } (C)ommon & (R)are & & (C)ommon & (R)are \\
\hline PC1+PC2 & .91 & .09 & & .90 & .10 \\
PR & .00 & 1.00 & & .13 & .87 \\
PC & .97 & .03 & & .90 & .10 \\
PC+PR & .58 & .42 & & .50 & .50 \\
PC1+PC2+PR & .88 & .12 & & .87 & .13 \\
\hline
\end{tabular}

Note - "PC" is a (P)erfectly predictive cue of the (C)ommon outcome, and "PR" is the (P)erfectly predictive cue of the (R)are outcome. The data for PC and PC + PR include two responses from each participant, because there were two different cues that were perfectly predictive of the common outcome - that is, PC1 and PC2. 
Table 3

Disjoint-Cues Category Structure for the

Learning Conditions in Experiment 2

\begin{tabular}{|c|c|c|c|c|c|c|}
\hline \multicolumn{6}{|c|}{ Cue } & \multirow[b]{2}{*}{ Outcome } \\
\hline $\mathrm{PC}_{0.5}$ & $\mathrm{PC}_{0.5}$ & $\mathrm{PR}_{1}$ & $\mathrm{PC}_{1}$ & $\mathrm{PR}_{0.5}$ & $\mathrm{PR}_{0.5}$ & \\
\hline 1 & 1 & 0 & 0 & 0 & 0 & $\mathrm{C}_{0.5}$ \\
\hline 1 & 1 & 0 & 0 & 0 & 0 & $\mathrm{C}_{0.5}$ \\
\hline 1 & 1 & 0 & 0 & 0 & 0 & $\mathrm{C}_{0.5}$ \\
\hline 0 & 0 & 1 & 0 & 0 & 0 & $\mathrm{R}_{1}$ \\
\hline 0 & 0 & 0 & 1 & 0 & 0 & $\mathrm{C}_{1}$ \\
\hline 0 & 0 & 0 & 1 & 0 & 0 & $\mathrm{C}_{1}$ \\
\hline 0 & 0 & 0 & 1 & 0 & 0 & $\mathrm{C}_{1}$ \\
\hline 0 & 0 & 0 & 0 & 1 & 1 & $\mathrm{R}_{0.5}$ \\
\hline
\end{tabular}

Note-This category structure is identical to the one used in Kruschke's (1996) Experiment 1, except for the critical difference that, here, the (C)ommon and (R)are outcomes did not share any cues. It is designated a "disjoint-cues" category structure, and the top four rows map onto the structure in the bottom of Figure 1. "PC" is a (P)erfectly predictive cue of a (C)ommon outcome, "PR" is the (P)erfectly predictive cue of a (R)are outcome, and 1 and 0 code whether a cue was present or absent, respectively, in the instance. A 0.5 subscript indicates a cue that always occurred with another cue during training and an outcome associated with two cues during training. A 1 subscript indicates a cue that always occurred alone during training and its corresponding outcome.

F, G, H or J.” Responses were made by pressing the corresponding keys on the computer keyboard. During the training phase, each response was followed by corrective feedback, which was either "CORRECT! This patient has disease [F, G, H, or J]" or "WRONG! This patient has disease [F, G, H, or J]." A wrong answer produced a tone. The participants were given up to $30 \mathrm{sec}$ to make a response. If they did not make a response in this time period, the message "FASTER!" appeared together with a tone. Following this was the instruction "After you have studied this case (up to 30 seconds), press the space bar to see the next one." If the participants did not press the space bar within this time period, an additional message appeared: "You have only 30 seconds to study each case." The next trial started automatically. There were 15 blocks of training with the eight instances from Table 3 per block ordered randomly.

Following the training phase, the participants were instructed that they would see a series of cases without feedback, some of which would have new combinations of symptoms or even no symptoms. They were instructed to respond on the basis of what they learned in the training phase and not just guess. The testing trials, all specified in the left column of Table 4, included various novel combinations of the previously learned symptoms, in addition to the four training instances. There were two testing blocks, each with all the listed trials. After each response, the message "Your diagnosis has been recorded" appeared. Pressing the space bar started the next trial.

The observational-learning condition was essentially identical to the feedback-learning condition, except that, in the training phase, the cues and correct outcome for a particular trial were displayed all at once. The participants were instructed to memorize the critical information, rather than making a response and being told the correct answer. They were required to study each case for a minimum of $1 \mathrm{sec}$ before a response prompt appeared telling them that they could study the case for up to $30 \mathrm{sec}$ before hitting the space bar to see the next case. The subsequent testing phase had all of the testing trial types from the feedback-learning condition plus five additional ones listed at the bottom of Table 4, including one where no cues were presented to directly test outcome base rate knowledge.

\section{Results and Discussion}

The testing trial response proportions for the decisionmaking and memorization conditions are shown in Table 2. There was no IBRE for PC+PR in either the decisionmaking $(\mathrm{C}=.58)$ or the memorization $(\mathrm{C}=.50)$ conditions. The decision-making condition produced signifi-

Table 4

Experiment 2: Testing Trial Response Proportions for the Feedback- and Observational-Learning Conditions

\begin{tabular}{|c|c|c|c|c|c|c|c|c|c|}
\hline \multirow[b]{2}{*}{ Symptoms } & \multirow{2}{*}{$\begin{array}{c}\text { Trials/ } \\
\text { Participant }\end{array}$} & \multicolumn{4}{|c|}{ Feedback Learning } & \multicolumn{4}{|c|}{ Observational Learning } \\
\hline & & $\mathrm{C}_{0.5}$ & $\mathrm{R}_{1}$ & $\mathrm{C}_{1}$ & $\mathrm{R}_{0.5}$ & $\mathrm{C}_{0.5}$ & $\mathrm{R}_{1}$ & $\mathrm{C}_{1}$ & $\mathrm{R}_{0.5}$ \\
\hline $\mathrm{PC}_{0.5}+\mathrm{PC}_{0.5}$ & 2 & .81 & .03 & .00 & .16 & .97 & .00 & .00 & .03 \\
\hline $\mathrm{PR}_{1}$ & 2 & .03 & .94 & .00 & .03 & .00 & .91 & .09 & .00 \\
\hline $\mathrm{PC}_{1}$ & 2 & .03 & .00 & .97 & .00 & .00 & .06 & .94 & .00 \\
\hline $\mathrm{PR}_{0.5}+\mathrm{PR}_{0.5}$ & 2 & .13 & .00 & .00 & .88 & .06 & .00 & .03 & .91 \\
\hline $\mathbf{P C}_{0.5}+\mathbf{P R}_{1}$ & 4 & .27 & .63 & .00 & .11 & .33 & .63 & .03 & .02 \\
\hline $\mathrm{PR}_{0.5}+\mathrm{PC}_{1}$ & 4 & .00 & .00 & .56 & .44 & .02 & .03 & .63 & .33 \\
\hline $\mathrm{PC}_{0.5}+\mathrm{PC}_{0.5}+\mathrm{PR}_{1}$ & 2 & .75 & .16 & .00 & .09 & .78 & .19 & .00 & .03 \\
\hline $\mathrm{PC}_{1}+\mathrm{PR}_{0.5}+\mathrm{PR}_{0.5}$ & 2 & .03 & .03 & .06 & .88 & .03 & .03 & .19 & .75 \\
\hline $\mathrm{PC}_{1}+\mathrm{PR}_{1}$ & 2 & .00 & .47 & .50 & .03 & .03 & .47 & .50 & .00 \\
\hline $\mathbf{P C}_{0.5}+\mathbf{P R}_{0.5}$ & 8 & .47 & .02 & .01 & .51 & .58 & .00 & .00 & .43 \\
\hline $\mathrm{PC}_{0.5}+\mathrm{PC}_{1}$ & 4 & .39 & .00 & .55 & .06 & .28 & .03 & .67 & .02 \\
\hline $\mathrm{PR}_{0.5}+\mathrm{PR}_{1}$ & 4 & .06 & .53 & .00 & .41 & .00 & .67 & .06 & .27 \\
\hline $\mathrm{PC}_{0.5}+\mathrm{PC}_{0.5}+\mathrm{PR}_{0.5}$ & 4 & .81 & .03 & .05 & .11 & .94 & .00 & .00 & .06 \\
\hline $\mathrm{PC}_{0.5}+\mathrm{PR}_{0.5}+\mathrm{PR}_{0.5}$ & 4 & .16 & .00 & .00 & .84 & .25 & .00 & .00 & .75 \\
\hline $\mathrm{PC}_{0.5}$ & 4 & .84 & .05 & .02 & .09 & .92 & .00 & .02 & .06 \\
\hline $\mathrm{PR}_{0.5}$ & 4 & .06 & .05 & .00 & .89 & .02 & .02 & .02 & .95 \\
\hline $\mathrm{PC}_{0.5}+\mathrm{PR}_{0.5}+\mathrm{PC}_{1}$ & 8 & - & - & - & - & .33 & .05 & .41 & .21 \\
\hline $\mathrm{PC}_{0.5}+\mathrm{PR}_{0.5}+\mathrm{PR}_{1}$ & 8 & - & - & - & - & .37 & .45 & .00 & .18 \\
\hline $\mathrm{PC}_{1}+\mathrm{PR}_{1}+\mathrm{PR}_{0.5}$ & 4 & - & - & - & - & .14 & .31 & .31 & .23 \\
\hline $\mathrm{PC}_{1}+\mathrm{PR}_{1}+\mathrm{PC}_{0.5}$ & 4 & - & - & - & - & .33 & .30 & .33 & .05 \\
\hline No cues & 2 & - & - & - & - & .31 & .22 & .31 & .16 \\
\hline
\end{tabular}

Note- "PC" is a (P)erfectly predictive cue for a (C)ommon outcome, and "PR" is a (P)erfectly predictive cue for a (R)are outcome. A 0.5 subscript indicates a cue that always occurred with another cue during training and an outcome associated with two cues during training. A 1 subscript indicates a cue that always occurred by itself during training and its corresponding outcome. The trials/participant column indicates the number of distinct but conceptually identical trials for each trial type. See the text for details. 
cantly more (C)ommon responses than in a standard IBRE task [.58 C proportion testing against $.35 \mathrm{C}$ from Kruschke $(1996$, Experiment 1$\left.\left.) ; \chi^{2}(2)=8.67, p<.013\right)\right]{ }^{2}$ and the memorization condition had a similar result $[.50>.35$; $\chi^{2}(2)=7.12, p<.028$; see note 2$]$.

These data indicate that asymmetric representation as a result of the disjoint-cues category structure is not by itself sufficient to produce an IBRE even though all of the evidence for (R)are (i.e., PR) was present in PC+PR, whereas only half of the evidence for (C)ommon was present (say, PC1, but not PC1+PC2). In particular, these results do not support Kruschke's (1996) explanation of the effect in terms of both asymmetric representation and explicit knowledge and moderated influence of the outcome base rates, especially since the results of Experiment 1 indicated responding consistent with the use of the summarized base rate information. At minimum, some other aspects of the standard IBRE task - such as trialby-trial learning, working memory constraints, and so forth - are needed, in addition to asymmetric representation, to produce the effect.

However, the conclusion that asymmetric representation is a necessary condition for the IBRE is somewhat supported by comparing these results with the results for the corresponding Experiment 1 conditions, which used the standard, shared-cue category structure: This disjointcues category structure resulted in significantly less influence of the explicitly presented base rate information for the decision-making condition on the critical case $\mathrm{PC}+\mathrm{PR}$ $\left[.94 \mathrm{C}>.58 \mathrm{C}\right.$ response proportion; $\chi^{2}(1) / 2=11.88 / 2=$ $5.94, p=.015$, where the division by 2 represents a conservative correction for the violation of independence in the present experiment resulting from the two test cases for PC+PR; Wickens, 1989, p. 390]. And the memorization condition had the same qualitative trend $[.67 \mathrm{vs}$. $.50 \mathrm{C}$ response proportions; $\chi^{2}(1)=1.71, p=.19$, but also violates independence]. These results are consistent with an asymmetric representation explanation of the IBRE if the explicitly presented frequency information overcame the greater similarity of $\mathrm{PC}+\mathrm{PR}$ to $\mathrm{PR} \rightarrow \mathrm{R}$ than to $\mathrm{PC} 1+\mathrm{PC} 2 \rightarrow \mathrm{C}$.

This indirect evidence for asymmetric representation as a necessary condition for the IBRE does not support a high-level-reasoning account of the effect, at least as per the theory of Juslin and colleagues (Juslin et al., 2001; Winman et al., 2005), since it assumes symmetric representation of the outcomes in the form of inference rules. In a looser sense, these results do not favor a high-levelreasoning account of the effect: Asymmetric representation should arguably facilitate an IBRE whether or not it is the reason for the effect in the standard learning task. So this facilitation, plus a hypothesized high-levelreasoning mechanism, might be expected to have produced a particularly strong IBRE in this condition, which it did not.

A comparison of the decision-making and memorization conditions in Experiments 1 and 2 suggests that memorizing the cue-outcome and base rate information reduced the incorporation of the base rates into the decision-making process, particularly for the critical trial
$\mathrm{PC}+\mathrm{PR}$. But even asymmetric representation of the two outcomes was not sufficient to overcome use of the summarized base rates and produce an IBRE. This suggests that the feedback- and observational-learning conditions would show even less competitive influence of the unsummarized outcome base rates with asymmetric representation and would produce a strong IBRE.

The response proportions for each testing trial type from the feedback- and observational-learning conditions are shown in Table 4. Many of the testing trials within each of the two testing blocks, although physically distinct, were conceptually identical in relation to the training instances. The number of equivalent trials per participant is specified in the "Trials/Participant" column in Table 4 for each trial type across the two testing blocks. For example, because the training instance $\mathrm{PC}_{0.5}+\mathrm{PC}_{0.5}$ had two physically different but conceptually identical cues (e.g., stuffy nose + back pain), the testing trial $\mathrm{PC}_{0.5}+\mathrm{PR}_{1}$ had two physically different but conceptually identical manifestations [e.g., stuffy nose $\left(\mathrm{PC}_{0.5}\right)+$ ear aches $\left(\mathrm{PR}_{1}\right)$ and back pain $\left(\mathrm{PC}_{0.5}\right)+$ ear aches $\left.\left(\mathrm{PR}_{1}\right)\right]$.

Overall, Table 4 shows that the results of the feedbackand observational-learning conditions were qualitatively comparable throughout. [Appendix B has the results of an observational-learning replication $(n=16)$ of the standard IBRE task (Figure 1, top), which were qualitatively comparable throughout to the results of Kruschke's (1996) Experiment 1.] Most important, feedback and observational learning resulted in significant and qualitative IBREs, as shown in bold in the fifth row of Table $4\left[\mathrm{C}_{0.5}=.27\right.$ and $\mathrm{C}_{0.5}=.33$ (Wilcoxon signed rank tests, $Z=-2.830, p=$ .004 , and $Z=-1.476, p=.14$; each data point was a participant's proportion of $\mathrm{C}_{0.5}$ responses out of the four trials]. This suggests that the hypothesized asymmetric representation of (C)ommon and (R)are outcomes is sufficient to account for the IBRE when the representations are acquired over a series of trials.

The presence of an IBRE in the feedback- and observational-learning conditions raises the critical question: What is the key difference between acquiring the asymmetric representation over a series of trials versus in summary form? The results for the $\mathrm{PC}_{1}+\mathrm{PR}_{1}$ and $\mathrm{PC}_{0.5}+\mathrm{PR}_{0.5}$ trials (Table 4 ) implicate base rate information. Both of these testing trials consisted of two cues that were perfectly correlated with their respective outcomes, occurred with the same number of additional cues, and differed only in the frequency of their associated outcomes during training. The response proportions for $\mathrm{PC}_{1}+\mathrm{PR}_{1}$ for both conditions were $\mathrm{C}_{1}=.50$ versus $\mathrm{R}_{1}=.47$, and for $\mathrm{PC}_{0.5}+\mathrm{PR}_{0.5}$ were, respectively, $\mathrm{C}_{0.5}=.47$ versus $\mathrm{R}_{0.5}=$ .51 and $\mathrm{C}_{0.5}=.58$ versus $\mathrm{R}_{0.5}=.43$. All of these results are consistent with profound base rate neglect and are based on two distinct trials of the first type and eight of the second. Note that it is not possible to have testing trials of this type using the standard category structure (Figure 1, top), where the critical test case $\mathrm{PC}+\mathrm{PR}$ is not a pure measure of base rate neglect. ${ }^{3}$ These results, together with the strong IBRE, suggest the following key difference between trialby-trial versus summary acquisition of the critical information: When the base rate information was only implicitly 
available over a series of trials, it was neglected at test; but when base rate information was explicitly summarized and available, it was used.

The third important set of results is for the test cases that are analogous to I+PC+PR from the standard, shared-cue category structure (Figure 1, top) and that have all of the cues for both a (C)ommon and a (R)are training instance. Specifically, the results for $\mathrm{PC}_{0.5}+\mathrm{PC}_{0.5}+\mathrm{PR}_{1}$, $\mathrm{C}_{0.5}=.75$ and $\mathrm{C}_{0.5}=.78$ for the feedback- and the observational-learning conditions, respectively, qualitatively replicated the (C)ommon responding for the standard learning task. However, the results for $\mathrm{PC}_{1}+\mathrm{PR}_{0.5}+\mathrm{PR}_{0.5}$ are not compatible with the strong application of base rate information in either learning condition, since (C) ommon responses in both conditions $\left(\mathrm{C}_{1}=.06\right.$ and $\mathrm{C}_{1}=$ .19 , respectively) were considerably less frequent than (R)are responses $\left(\mathrm{R}_{0.5}=.88\right.$ and $\mathrm{R}_{0.5}=.75$, respectively), despite the fact that all the cues associated with both outcomes were present.

Finally, the no-cues (symptoms unavailable) trials from the observational-learning condition (Table 4, bottom; not included in the feedback-learning condition) indicate that the participants had some knowledge of the outcome base rates: There was a qualitative trend for more (C)ommon responses than for $(\mathrm{R})$ are ones $[\mathrm{C}=.62$ and $\mathrm{R}=.38$; $\left.\chi^{2}(3)=2.25, p=.52\right]$.

Kruschke's (1996) account of the IBRE assumes asymmetric representation of the (C)ommon and (R)are outcomes as a result of error-driven learning and explicit base rate knowledge, which has a moderating influence on decision making during the testing phase. The observational-learning condition does not imply the need for error-driven learning, although it might be argued that the participants implicitly generated an error signal despite not overtly producing a response. More important, Kruschke's (1996) evidence for base rate knowledge was that participants rated the (C)ommon outcome as significantly more frequent than the (R)are one when explicitly asked to do so after completing the testing phase, although their ratings of slightly above $2: 1(\mathrm{C}: \mathrm{R})$ were still somewhat less than the actual values of $3: 1$. But the evidence for profound base rate neglect in the two learning conditions here is not consistent with this account. Although it is entirely possible that people had base rate knowledge, implied at least by the less direct measure provided by the no-cue test case, the overall results suggest that this knowledge was not systematically applied and had little if any moderating influence on the occurrence of the IBRE.

Juslin et al.'s (2001) account of the IBRE assumes symmetric representation of the $(\mathrm{C})$ ommon and $(\mathrm{R})$ are outcomes and a high-level-reasoning procedure involving eliminative inference. Kruschke (2001) argued that this theory predicts an IBRE for a learning task that encourages symmetric representation of the (C)ommon and (R)are outcomes as a result of the absence of a shared, (I)mperfectly predictive cue. However, his new experiment did not result in an IBRE under these conditions. Likewise, in the present experiment, the outcomes $\mathrm{C}_{0.5}$ and $\mathrm{R}_{0.5}$ were likely to be symmetrically encoded because they corresponded to $\mathrm{PC}_{0.5}+\mathrm{PC}_{0.5}$ and $\mathrm{PR}_{0.5}+\mathrm{PR}_{0.5}$, respectively, during learning. However, as converging evidence for Kruschke's (2001) critique, no IBRE occurred at test for $\mathrm{PC}_{0.5}+\mathrm{PR}_{0.5}$.

In summary, this experiment suggests that forced asymmetric representation in a trial-by-trial learning task, but not in a summary decision-making task, is sufficient to produce the IBRE. In addition, clear evidence of profound base rate neglect was found in the presence of the effect. This supports the hypothesis, evaluated in Experiment 3, that asymmetric representation and systematic base rate neglect are individually necessary and jointly sufficient to account for the effect.

\section{EXPERIMENT 3 \\ Evaluating Combinations of Representation and Decision Making}

The previous experiments had two distinct methodologies, summary decision making or trial-by-trial learning, but used the same cue-outcome and base rate information. The summary decision-making conditions did not result in the IBRE, even when using the disjoint-cues category structure matching hypothesized asymmetric representation, whereas the learning conditions did result in the effect, like the standard task, even without feedback.

Experiment 2 suggested that the key difference between the summary decision-making and trial-by-trial learning conditions was the status of the base rate information: In the summary decision-making conditions, the outcome frequency information was explicitly summarized, and so, arguably, used, resulting in no IBRE. In the trial-by-trial learning conditions, the base rate information was only implicitly available over a series of trials, and so, arguably, was not used, resulting in the IBRE. The observationallearning condition in Experiment 2 indicated some base rate knowledge, as shown by no-cue trials, and Kruschke (1996) demonstrated that participants had some base rate knowledge when explicitly asked in a posttest. However, this base rate information plausibly had less influence on responding when more implicitly acquired, because it may not have been so constantly active - hence, the hypothesis that asymmetric representation and base rate neglect are individually necessary and jointly sufficient for the effect.

This experiment used a methodology intermediate between summary decision making and trial-by-trial learning in an attempt to find the simplest possible conditions for the IBRE. The methodology was to take part of the explicitly summarized information from the previous decision-making conditions and make it more implicit in the form of a short list of instances, rather than an explicit summary. For the base rate information, instead of giving the explicit summary, "approximately three times as many patients have had terrigitis as have had coralgia," the participants were given a short list of patients consistent with the base rates: "Patient 1 had terrigitis, Patient 2 had terrigitis, Patient 3 had coralgia, Patient 4 had terrigitis ...." For cue-outcome information, instead of the explicit summary, "Patients with the combination of symptoms sore muscles and dizziness have always had 
terrigitis ...," the participants were given a short list of patients, "Patient 1 had the symptoms sore muscles and dizziness. This patient had terrigitis. Patient 2 had the symptoms dizziness and sore muscles. This patient had terrigitis. Patient 3 had the symptoms stuffy nose and dizziness. This patient had coralgia ...."

This experiment used the listing methodology to make either just the base rate information or both the cueoutcome and the base rate information more implicit in the context of either the disjoint-cues (Figure 1, bottom) or the standard, shared-cue (Figure 1, top) category structures. The purpose of the resulting four conditions, shown in simplified form in Table 5, was to check which combinations of summarized or listed information and standard, shared-cue, or disjoint-cues category structures were jointly sufficient to produce the IBRE.

In relation to prior conditions, the summarized disjoint-cues and summarized shared-cue conditions were essentially identical to the pure decision-making conditions in Experiments 2 and 1, respectively, except that, here, the outcome base rate information was listed rather than summarized. The listed disjoint-cues condition was similar to the observational-learning condition in Experiment 2, except that the cue-outcome and base rate information were presented all at once as a short list, rather than over a series of trials. The listed shared-cue condition was similar to the standard IBRE task, except for the list format and lack of feedback. Finally, the summarized disjoint-cues and listed shared-cue conditions had a posttest base rate judgment question, followed by a second testing phase to evaluate the activation of base rate information on the occurrence of the effect. Again, see Table 5's simplified condition summary.

\section{Method}

Participants. The summarized disjoint-cues, summarized shared-cue, listed disjoint-cues, and listed shared-cue conditions had 30,20,30, and 31 unpaid $^{4}$ participants, respectively, mainly psychology students at UCL.

Materials and Procedure. The materials and procedure were similar to those for the decision-making conditions in Experiments 1 and 2 in terms of cover story, instructions, and use of two versions based on different symptom and disease labels. The key difference was that the summary of the outcome base rates was replaced by a quasirandom list of 12 patients, with their diseases corresponding to the outcome base rates of $3: 1(\mathrm{C}: \mathrm{R})$. The instructions, list, and initial testing trials all occurred on one page.

The four conditions, summarized in Table 5, presented the cueoutcome information as either a list of instances or a summary matching either the shared-cue (Figure 1 top) or the disjoint-cues (Figure 1, bottom) category structures, resulting in summarized disjoint-cues, summarized shared-cue, listed disjoint-cues, and listed shared-cue conditions. Again, note that all the conditions presented the outcome base rate information in the form of a list and differed only in whether the cue-outcome information was also in the list or just explicitly summarized.

After the initial test page, the participants in the summarized disjoint-cues and listed shared-cue conditions were asked to recall the base rates for the two diseases by indicating how many patients out of 12 had each disease, without looking back at the previous page. This was followed by another set of the same testing trials in a different order. The last testing trial of the second test phase was a completely new symptom. During the second testing phase, the participants in the summarized disjoint-cues condition had the summarized cue-outcome relations again made available to them, but not the listed outcome base rate information, whereas the participants in the listed shared-cue condition again had the initial list, because there was no summary.

\section{Results and Discussion}

Table 6 shows the test trial response proportions for all four conditions (also see Table 5), with the critical case

Table 5

Abstract Form of the Information Presented in Each of the Four Conditions in Experiment 3

\begin{tabular}{|c|c|c|c|c|}
\hline \multirow{2}{*}{$\begin{array}{l}\text { Category } \\
\text { Structure }\end{array}$} & \multicolumn{4}{|c|}{ Cue/Outcome Information } \\
\hline & \multicolumn{2}{|c|}{ Summarized } & \multirow{2}{*}{\multicolumn{2}{|c|}{$\begin{array}{c}\text { Listed } \\
\text { No summary }\end{array}$}} \\
\hline \multirow[t]{8}{*}{ Disjoint cues } & Summary & $\begin{aligned} & \mathrm{PC} 1+\mathrm{PC} 2 \rightarrow \mathrm{C} \\
& \mathrm{PR} \rightarrow \mathrm{R}\end{aligned}$ & & \\
\hline & List : & $\mathrm{C}$ & List: & $\mathrm{PC} 1+\mathrm{PC} 2 \rightarrow \mathrm{C}$ \\
\hline & & $\mathrm{C}$ & & $\mathrm{PC} 2+\mathrm{PC} 1 \rightarrow \mathrm{C}$ \\
\hline & & $\mathrm{R}$ & & $\mathrm{PR} \rightarrow \mathrm{R}$ \\
\hline & & $\mathrm{C}$ & & $\mathrm{PC} 1+\mathrm{PC} 2 \rightarrow \mathrm{C}$ \\
\hline & & . & & . \\
\hline & & . & & . \\
\hline & & $\cdot$ & & · \\
\hline \multirow[t]{9}{*}{ Shared cue } & Summary & $\mathrm{I}+\mathrm{PC} \rightarrow \mathrm{C}$ & & No summary \\
\hline & & $\mathrm{I}+\mathrm{PR} \rightarrow \mathrm{R}$ & & \\
\hline & List: & $\mathrm{C}$ & List: & $\mathrm{PC}+\mathrm{I} \rightarrow \mathrm{C}$ \\
\hline & & $\mathrm{C}$ & & $\mathrm{I}+\mathrm{PC} \rightarrow \mathrm{C}$ \\
\hline & & $\mathrm{R}$ & & $\mathrm{PR}+\mathrm{I} \rightarrow \mathrm{R}$ \\
\hline & & $\mathrm{C}$ & & $\mathrm{PC}+\mathrm{I} \rightarrow \mathrm{C}$ \\
\hline & & . & & . \\
\hline & & . & & . \\
\hline & & . & & . \\
\hline
\end{tabular}

Note_- "PC" refers to a (P)erfectly predictive cue of a (C)ommon outcome, "PR" to a (P)erfectly predictive cue of a (R)are outcome, and "I" to an (I)mperfectly predictive/shared cue. In the disjoint-cues conditions, PC1 and PC2 were different symptoms. See the main text for detailed specification of summary and list information. 
$\mathrm{PC}+\mathrm{PR}$ in bold. The first test phase of the summarized disjoint-cues condition resulted in a significant IBRE $(.32 \mathrm{C}$ response proportion $<.5$; sign test, $p=.035$, where the data point for each participant was the proportion of (C)ommon responses on the two different tests of PC + PR). Subsequently, the participants' outcome base rate judgments indicated a significantly higher rating of the $(\mathrm{C})$ ommon outcome than the $(\mathrm{R})$ are one $[.64 \mathrm{C}>.50$; $t(29)=6.51, p<.001]$. After the base rate judgment, there was no longer a significant IBRE $(.47 \mathrm{C} \sim .50$; sign test, $p=.84$ ), and the effect was significantly reduced as compared with the results from before the base rate judgment $(.32 \mathrm{C}<.47 \mathrm{C}$; Wilcoxon signed ranks test, $Z=-2.31, p=.021$, based on the difference of the $\mathrm{C}$ response proportions for the two test phases).

This condition indicates that the outcome-listing methodology was effective in reducing the impact of base rate knowledge on the critical transfer trial PC + PR: There was a significant IBRE for the first testing phase of this condition while there was not a significant IBRE for the decision-making condition from Experiment 2, which differed only in that the outcomes were explicitly summarized in that condition rather than listed, as in the present condition. However, the participants in the present experiment did exhibit significant, although imperfect, base rate knowledge that was, nevertheless, strongly neglected on the first critical test trial, but activating this knowledge by the rating question was sufficient to significantly reduce the magnitude of the effect. Overall, this condition supports the hypothesis that asymmetric representation and base rate neglect are jointly sufficient to produce the IBRE.

The summarized shared-cue condition did not result in an $\mathrm{IBRE}^{5}$ for $\mathrm{PC}+\mathrm{PR}[.65 \mathrm{C}$ response proportion $>.35 \mathrm{C}$
(Kruschke, 1996), $\chi^{2}(1)=5.30, p=.02$, and $.65 \mathrm{C} \sim>.5$, $\left.\chi^{2}(1)=1.80, p=.18\right]$. In addition, these results indicate significantly less base-rate-consistent responding for $\mathrm{PC}+\mathrm{PR}$ than do the results of the pure decision-making condition in Experiment 1, where the base rates were explicitly summarized $\left[.65 \mathrm{C}<.94 \mathrm{C} ; \chi^{2}(1)=7.40, p=.007\right]$.

The results of this condition suggest that base rate neglect from the listing methodology was not by itself sufficient to produce the IBRE. Arguably, asymmetric representation is necessary, although the listing methodology was again shown to reduce the influence of the base rates, as compared with the base rate summary in the decisionmaking condition in Experiment 1.

The listed disjoint-cues condition resulted in a significant IBRE for PC + PR $(.30 \mathrm{C}<.50$; sign test, $p=.017$, based on the $\mathrm{C}$ response proportion from the two equivalent trials for each participant). Also, the other data points are qualitatively similar to Experiment 2's feedback-learning condition. These results again indicate that asymmetric representation and base rate neglect are jointly sufficient to account for the effect.

Finally, the listed shared-cue condition resulted in a significant IBRE for PC+PR in the first test phase [.26 $\mathrm{C}<$ $\left..50, \chi^{2}(1)=7.26, p=.007\right]$. Subsequently, the participants' outcome base rate estimates indicated a significantly higher rating of the $(\mathrm{C})$ ommon than of the $(\mathrm{R})$ are outcomes $[\mathrm{C}=.68>.50 ; t(30)=7.10, p<.001]$. After the base rate judgment, the magnitude of the IBRE in the second testing phase was reduced relative to the first phase [to $.45 \mathrm{C}$ from $.26 \mathrm{C} ; \chi^{2}(1)=2.54, p=.11$ ] and was itself no longer significantly different from $.5\left[\chi^{2}(1)=0.29\right.$, $p=.59]$. The remaining test trials were highly comparable to those in the standard learning task (e.g., Kruschke, 1996; our Table 1, in parentheses).

Table 6

Experiment 3: Testing Trial Response Proportions for All Four Conditions

\begin{tabular}{|c|c|c|c|c|c|c|c|c|c|}
\hline \multirow{4}{*}{$\begin{array}{l}\text { Category } \\
\text { Structure }\end{array}$} & \multirow[b]{4}{*}{ Cues/Outcomes } & \multicolumn{8}{|c|}{ Cue/Outcome Information } \\
\hline & & \multicolumn{4}{|c|}{ Summarized } & \multicolumn{4}{|c|}{ Listed } \\
\hline & & \multicolumn{2}{|c|}{ Phase 1} & \multicolumn{2}{|c|}{ Phase 2} & \multicolumn{2}{|c|}{ Phase 1} & \multicolumn{2}{|c|}{ Phase 2} \\
\hline & & $\mathrm{C}$ & $\mathrm{R}$ & $\mathrm{C}$ & $\mathrm{R}$ & $\mathrm{C}$ & $\mathrm{R}$ & $\mathrm{C}$ & $\mathrm{R}$ \\
\hline \multirow[t]{6}{*}{ Disjoint cues } & $\mathrm{PC}+\mathrm{PC}$ & .97 & .03 & 1.00 & .00 & .97 & .03 & - & - \\
\hline & PR & .03 & .97 & .03 & .97 & .00 & 1.00 & - & - \\
\hline & $\mathrm{PC}$ & 1.00 & .00 & .97 & .03 & .93 & .07 & - & - \\
\hline & $\mathbf{P C}+\mathbf{P R}$ & .32 & .68 & .47 & .53 & .30 & .70 & - & - \\
\hline & $\mathrm{PC}+\mathrm{PC}+\mathrm{PR}$ & .87 & .13 & .90 & .10 & .97 & .03 & - & - \\
\hline & Novel cue & - & - & .17 & .83 & - & - & - & - \\
\hline \multirow[t]{8}{*}{ Shared cue } & $\mathrm{PC}+\mathrm{I}$ & 1.00 & .00 & - & - & 1.00 & .00 & .94 & .07 \\
\hline & $\mathrm{PR}+\mathrm{I}$ & .21 & .79 & - & - & .03 & .97 & .10 & .90 \\
\hline & I & .70 & .30 & - & - & .90 & .10 & .77 & .23 \\
\hline & $\mathrm{PC}$ & .90 & .10 & - & - & .90 & .10 & .94 & .07 \\
\hline & PR & .05 & .95 & - & - & .00 & 1.00 & .07 & .94 \\
\hline & $\mathbf{P C}+\mathbf{P R}$ & .65 & .35 & - & - & .26 & .74 & .45 & .55 \\
\hline & $\mathrm{I}+\mathrm{PC}+\mathrm{PR}$ & .70 & .30 & - & - & .61 & .39 & .55 & .45 \\
\hline & Novel cue & - & - & - & - & - & - & .29 & .71 \\
\hline
\end{tabular}

Note- "PC" refers to a (P)erfectly predictive cue of a (C)ommon outcome, "PR" to a (P)erfectly predictive cue of a (R)are outcome, and "I" to an (I)mperfectly predictive/shared cue. In the disjoint-cues conditions, the data for $\mathrm{PC}$ and $\mathrm{PC}+\mathrm{PR}$ include two responses from each participant, because there were two different symptoms, PC1 and PC2, that were (P)erfectly predictive of the (C)ommon outcome. "Phase 1" and "Phase 2 " refer to the testing results from before and after the base rate knowledge evaluation question. See the main text for details. 
Overall, this last condition suggests that simultaneously presenting a short list of cases captures the essential aspects of the standard trial-by-trial learning and feedback task in terms of producing the IBRE. Response generation, feedback, and interaction with working memory constraints do not appear to be necessary conditions. In particular, a comparison with the summarized shared-cue condition suggests that listing, rather than summarizing, the cue-outcome information is a necessary condition for the effect. The presentation of a list, rather than a summary, seems more likely to facilitate the hypothesized asymmetric representation.

These results are consistent with the hypothesis that strong base rate neglect is a necessary condition for the IBRE: The participants demonstrated base rate knowledge when asked, but the Phase 2 test results indicate that knowledge was unlikely to be impacting responding to the critical test PC + PR in Phase 1, contrary to the moderating influence of explicit base rate knowledge on test case responding hypothesized by Kruschke (1996). Furthermore, these results provide an explanation of his evidence for base rate knowledge from a posttest question to rate the outcome base rates: Activating the base rate knowledge by direct inquiry led, in our second testing phase, to a significant reduction in the IBRE, suggesting that asymmetric representation by itself was not sufficient to overcome the newly activated base rate knowledge and produce a robust effect.

Furthermore, these results do not implicate working memory limitations as a necessary condition for the IBRE. This does not particularly support Krushke's (1996) account of the effect in terms of error-driven selective attention or Juslin et al.'s (2001) account in terms of selective activation of inference rules, since both invoke capacity limitations.

We believe Juslin et al.'s (2001) eliminative inference account as formalized in the ELMO model has been shown by Kruschke (2001) and several of our experiments to be an inadequate explanation of the IBRE. However, the novel cue trials for this last condition and for the summarized disjoint-cues condition (Table 6) replicate the novel symptom effect predicted by Juslin et al.: (R)are responding to a novel cue at test. As they pointed out, this result is not predicted by Kruschke's (1996) ADIT model: Since participants receive no feedback on a novel cue during training, ADIT predicts base-rate-consistent responding to a novel cue at test. However, as was mentioned by Medin and Edelson (1988), the combination of three cues, I+PC+PR, is also novel. Furthermore, a new cue is novel in a different way than PC+PR, which is a novel combination of old cues. Although this novel cue result is puzzling, it does not by itself seem to provide an adequate account of the effect, although it does suggest high-level mechanisms.

Finally, it might be argued that although this condition produced an IBRE, it did so for reasons that are different than those for the IBRE. But this seems implausible, given its systematic and conceptual relationship to Experiments 1 and 2. Furthermore, even if the reasons for the IBRE here were different from those in the standard task, the results are still puzzling and, hence, interesting for essentially the same reasons. Finally, in the absence of clear contrary evidence, parsimony suggests assuming the same mechanisms, since the cue-outcome and base rate information were essentially the same as those in the standard IBRE task.

\section{GENERAL DISCUSSION}

The purpose of this research was to evaluate the necessary and sufficient conditions for the inverse base rate effect (IBRE) in human category learning by exploring systematic deviations from the standard IBRE learning task (e.g., Medin \& Edelson, 1988), which produces this puzzling effect: low-frequency-outcome responding to a pair of otherwise perfectly conflicting cues after a cueoutcome feedback learning phase (Figure 1, top). Almost all research on the IBRE has hypothesized it to be the result of either asymmetric outcome representations formed during learning or some aspect of decision making at test (see the introduction). Experiment 1's pure decisionmaking condition was based on an explicit summary of the cue-outcome and outcome base rate information from the standard learning task and did not result in the effect. As is summarized in Table 7, the remaining experimental conditions were systematic deviations between this condition and the standard feedback learning task.

In Table 7, the "Std" condition is the standard learning task and has the following variable values: The cueoutcome and base rate information were only implicitly available over trials, rather than explicitly summarized; the critical information was memorized, rather than explicitly available; the information was presented over trials, rather than simultaneously; the category structure was the shared-cue structure (Figure 1, top), rather than the disjoint-cues structure (Figure 1, bottom); corrective feedback was given on each learning trial; and the IBRE occurred. Experiment 1's pure decision-making condition (1DM in Table 7) had contrasting values on all of these variables except for the category structure, and the remaining conditions had contrasting variables between these two (see Table 7's caption for experimental condition abbreviations).

\section{Asymmetric Representation and Base Rate Neglect As Necessary and Sufficient Conditions}

If asymmetric representation is necessary, the IBRE should not occur in the absence of conditions that facilitate the asymmetry but should occur in the presence of those conditions if all other necessary conditions are also present - namely, base rate neglect. A similar argument applies to base rate neglect.

As compared with prior research on the IBRE, Experiment 2 provided a more direct evaluation of the asymmetric representation that has been widely hypothesized as a necessary condition for the IBRE. Its conditions were based directly on the disjoint-cues category structure (Figure 1, bottom) designed to approximate the hypothesized asymmetric representation formed in the standard learning task, although participants and theoretical formulations are somewhat variable (see the introduction). Nevertheless, if asymmetric representation is a necessary condition for the 
Table 7

Summary of Manipulated Variables and Outcome for All Experiments

\begin{tabular}{|c|c|c|c|c|c|c|c|c|c|c|c|c|c|c|}
\hline \multirow[b]{2}{*}{ Condition } & \multicolumn{2}{|c|}{$\begin{array}{l}\text { Cue-Outcome } \\
\text { Information }\end{array}$} & \multicolumn{2}{|c|}{$\begin{array}{c}\text { Base Rate } \\
\text { Information }\end{array}$} & \multicolumn{2}{|c|}{ Memorized? } & \multicolumn{2}{|c|}{ Information Presented } & \multicolumn{2}{|c|}{$\begin{array}{l}\text { Category } \\
\text { Structure }\end{array}$} & \multicolumn{2}{|c|}{ Feedback? } & \multicolumn{2}{|c|}{ IBR Effect } \\
\hline & Explicit & Implicit & Explicit & Implicit & Yes & No & Simultaneously & Trial by Trial & Shared & Disjoint & Yes & No & Present & Absent \\
\hline Std & & $*$ & & $*$ & $*$ & & & $*$ & $*$ & & $*$ & & $*$ & \\
\hline $1 \mathrm{DM}$ & $*$ & & $*$ & & & * & $*$ & & * & & & $*$ & & $*$ \\
\hline 1MEM & $*$ & & $*$ & & $*$ & & $*$ & & * & & & $*$ & & $*$ \\
\hline $2 \mathrm{DM}$ & $*$ & & $*$ & & & * & $*$ & & & * & & $*$ & & $*$ \\
\hline 2MEM & $*$ & & * & & $*$ & & $*$ & & & $*$ & & $*$ & & $*$ \\
\hline $2 \mathrm{FL}$ & & * & & * & $*$ & & & $*$ & & $*$ & $*$ & & $*$ & \\
\hline $2 \mathrm{OL}$ & & * & & $*$ & $*$ & & & $*$ & & $*$ & & $*$ & * & \\
\hline $3 \mathrm{SD}$ & $*$ & & & $*$ & & * & $*$ & & & $*$ & & $*$ & $*$ & \\
\hline $3 \mathrm{SS}$ & $*$ & & & $*$ & & $*$ & $*$ & & * & & & $*$ & & $*$ \\
\hline $3 \mathrm{LD}$ & & $*$ & & $*$ & & $*$ & $*$ & & & $*$ & & * & $*$ & \\
\hline $3 \mathrm{LS}$ & & $*$ & & $*$ & & $*$ & $*$ & & * & & & $*$ & $*$ & \\
\hline
\end{tabular}

Note- "Std" refers to the standard inverse base rate (IBR) experiment as in Medin and Edelson (1988), Kruschke (1996), and so forth; "1DM" = Experiment 1's decision-making condition; "1MEM" = Experiment 1's memorization condition; "2FL" and "2OL" = feedback- and observationallearning conditions in Experiment 2; "3SD," "3SS," "3LD," and "3LS" = the summarized disjoint-cues, summarized shared-cue, listed disjoint-cues, and listed shared-cue conditions, respectively, in Experiment 3 (Table 5).

effect, the disjoint-cues category structure (Figure 1, bottom) should result in a particularly robust IBRE when in conjunction with base rate neglect (discussed below). This was found in Experiment 2's feedback-learning (2FL in Table 7) and observational-learning (2OL) conditions and Experiment 3's summarized disjoint-cues (3SD) and listed disjoint-cues (3LD) conditions in the putative presence of the additional necessary condition, induced base rate neglect.

We have argued that the summary decision-making tasks on the standard shared-cue category structure (Figure 1, top), Experiment 1 (1DM and 1MEM in Table 7), and Experiment 3's summarized shared-cue condition (3SS) did not result in the IBRE because the cue-outcome information was explicitly summarized. So there was no motivation to produce a representation that was different from that summary, such as an asymmetric one. Correspondingly, the presence of the effect in Experiment 3's listed shared-cue condition (3LS) suggests that the lack of an explicit summary was enough to induce asymmetric representation. Hence, the absence or presence of the effect in these results corresponds, in conjunction with base rate neglect, to whether the cue-outcome relations were explicitly summarized or available only in a more implicit format (Table 7). Consequently, these results provide evidence that asymmetric representation is necessary, because the IBRE did not occur in the absence of the conditions argued to facilitate asymmetric representation (1DM, 1MEM, and 3SS in Table 7) but always occurred in the presence of those conditions when conditions argued to facilitate base rate neglect were also present (2FL, 2OL, $3 \mathrm{SD}, 3 \mathrm{LD}$, and $3 \mathrm{LS}$ in Table 7). Taken together, these results suggest that asymmetric representation is a sufficient condition for the IBRE when occurring together with base rate neglect, although not by itself (2DM and 2MEM)0.

In addition, our results suggest that participants have but do not systematically apply outcome base rate knowledge at test in the standard task. As such, base rate neglect is a necessary condition that is jointly sufficient with asymmetric representation to produce the effect. The influence of base rates was assessed in three ways.
First, evidence for base rate neglect came via direct test trial assessment in Experiment 2's feedback- and observational-learning conditions (2FL and 2OL in Table 7) based on the disjoint-cues category structure (Figure 1, bottom). These conditions provided strong evidence of profound base rate neglect $\left(\mathrm{PC}_{1}+\mathrm{PR}_{1}\right.$ and $\mathrm{PC}_{0.5}+\mathrm{PR}_{0.5}$ in Table 4) independently of the representation asymmetry evaluation provided by the critical test case $\left(\mathrm{PC}_{0.5}+\mathrm{PR}_{1}\right.$ in Table 4$)$.

Second, an indirect assessment of base rate knowledge occurred in the decision-making and memorization conditions in Experiments 1 and 2 (1DM, 1MEM, 2DM, and 2MEM in Table 7) and an indirect assessment of base rate neglect occurred in Experiment 3 (3SD, 3SS, 3LD, and $3 \mathrm{LS}$ ) via the critical test case PC+PR, which also defines the presence or absence of the IBRE. This indirect assessment was in the context of experimental manipulations that, arguably, encouraged or discouraged base rate neglect. In conditions 1DM, 1MEM, 2DM, and 2MEM, the base rate information was summarized and so, arguably, used because it was explicitly available. None of these conditions resulted in the effect. However, in Experiment 3 , the outcome base rate information was not summarized but was only implicitly available as a list. All the conditions resulted in the effect except for 3SS, which still showed evidence of reduced base rate use.

Third, the direct rating of the base rates in Experiment 3 (3SD and 3LS in Table 7) showed that getting the participants to explicitly summarize their base rate knowledge dramatically reduced the IBRE in a second testing phase. This implies that an explicit summary of the base rates reduced base rate neglect, since nothing other than the base rate summary question occurred between the two testing phases. So base rate neglect is a likely necessary condition, since a manipulation to reduce it also reduced the IBRE.

Together, these results imply that base rate neglect is necessary for the effect, since it did not occur in the absence of conditions likely to induce base rate neglect. Furthermore, it is jointly sufficient with asymmetric representation, since the effect always occurred in the presence of conditions argued to induce asymmetric representation and 
base rate neglect. Finally, the occurrence of the inverse base rate effect even with the simple listing methodology used in Experiment 3 implies that other aspects of the standard learning task, such as response generation and corrective feedback or working memory load, are not necessary.

\section{Relating Prior Research to Future Research}

Even if asymmetric outcome representation and base rate neglect are the causes of the IBRE, the reasons for these conditions are themselves far from clear. It is worth reconsidering the two distinct theoretical perspectives that prior research on the IBRE can be grouped into, currently represented by Kruschke $(1996,2001,2003)$ and Juslin and colleagues (Juslin et al., 2001; Winman et al., 2003, 2005). These two perspectives correspond quite closely to the broad theoretical distinction between low-level associative and high-level rule-based processing: Kruschke's (1996, 2001, 2003) explanation of the IBRE is basically a low-level account with incremental associative learning moderated by error-driven selective attention. In particular, his systematic base rate influence hypothesis is formalized in his EXIT model via the same low-level associative mechanisms. In contrast, Juslin and colleagues (Juslin et al., 2001; Winman et al., 2003, 2005) have emphasized high-level processes, including the formation of rules and use of sophisticated eliminative inference in the context of novelty effect reasoning.

Both of the specific instantiations for these two perspectives (Juslin et al., 2001; Kruschke, 1996) have aspects that are incompatible with the research presented here and elsewhere. But research has not eliminated either of these broad perspectives on the IBRE, and both are conceptually compatible with asymmetric representation and base rate neglect: Rules can be specified to match almost any representation formed through associative learning, and base rate knowledge need not be a fundamental part of either associative or rule-based representations. For example, Kruschke, Kappenman, and Hetrick (2005) found eyetracking evidence for asymmetric representation in an experimental design related to the IBRE category structure but still accounted for the results with an extension of the ADIT model (EXIT, mentioned above).

Even if rule representation is limited to simple rules, differentiating high-level inference rules from low-level associations is not necessarily straightforward. For example, Medin and Edelson's (1988) Experiment 4 showed a particularly strong IBRE after participants were instructed to search for a perfectly predictive cue for each outcome. This result seems more compatible with the high-level formation of rules than with low-level association formation, but a low-level associative framework can probably be stretched to account for this by arguing that this manipulation more strongly focused selective attention. Likewise, Winman et al. (2005) argued for the importance of rule formation by showing that between-subjects variability in the IBRE task correlates with evidence of rule formation in a second task (Shanks \& Darby, 1998) designed to assess rule-based versus similarity-based processing. Perhaps some combination of representation and selective attention could explain this result in a low-level associative framework. But more important, Juslin et al. (2001) did not translate this correlation into a precise specification of rules in the IBRE task, leaving open the possibility that the correlation is the result of aspects common to both high- and low-level perspectives.

Given the current prevalence of category-learning research emphasizing the distinction between high-level rulebased and low-level association-/similarity-based representation (e.g., Ashby, Alfonso-Reese, Turken, \& Waldron, 1998; Erickson \& Kruschke, 1998), this issue seems particularly relevant in the context of the IBRE. One way to distinguish high-level and low-level explanations of the effect might be to use a dual-task environment designed to tax high-level processes more than low-level ones - for example, performing an observational-learning version of the IBRE task while doing a concurrent working memory task. Another way to distinguish high-level and low-level accounts might be with some form of brain imaging - for example, duplicating Winman et al.'s (2005) experiment and comparing the results from the IBRE task with those for the Shanks and Darby (1998) task and prior results implicating different kinds of processing. Yet another way to possibly distinguish these perspectives would be to more thoroughly explore whether or not animals exhibit the IBRE (see Fagot, Kruschke, Dépy, \& Vauclair, 1998, for consideration of a related task).

Finally, from a more general perspective, the IBRE is a specific case for studying how humans handle the inductive conflict that occurs when making classifications on the basis of ambiguous or conflicting information, and it is particularly relevant because real-world categories differ enormously in their base rates. Biases are a widely accepted aspect of human decision making, but the nature of these biases, particularly in category learning, is still being elucidated. A better understanding of biases is likely to be important for explaining how humans find adaptive categories in the much larger space of possible, arbitrary categories.

In conclusion, through this research, we have gained a deeper understanding of the role of base rate knowledge and representational asymmetry in category learning. Specifically, individuals (1) can reveal striking failures to deploy base rate information and (2) can form asymmetric representations when confronted with differentially diagnostic information. These effects combine to generate the IBRE but, more important, are likely to affect performance in category learning generally. A full understanding of the precise causes of these effects is not provided by current theories, but they emphasize important questions about basic issues, such as the role of inductive biases in the presence of inductive conflict and rule-based versus similarity-based and high-level versus low-level processing in learning.

\section{AUTHOR NOTE}

This research was supported by Grant RES-000-23-0024 from the United Kingdom Economic and Social Research Council. We thank John Kruschke, Justin Savage, and three anonymous reviewers for comments and suggestions on a previous version of the manuscript. Correspondence concerning this article should be addressed to M. K. Johansen, School of Psychology, Cardiff University, Tower Building, Park Place, Cardiff CF10 3AT, Wales (e-mail: johansenm@cardiff.ac.uk). 


\section{REFERENCES}

ANDERSON, J. R. (1990). The adaptive character of thought. Hillsdale, NJ: Erlbaum.

Ashby, F. G., Alfonso-Reese, L. A., Turken, A. U., \& Waldron, E. M. (1998). A neuropsychological theory of multiple systems in category learning. Psychological Review, 105, 442-481.

Binder, A., \& Estes, W. K. (1966). Transfer of response in visual recognition situations as a function of frequency values. Psychological Monographs: General \& Applied, 80, 1-26.

Bohil, C. J., Markman, A. B., \& Maddox, W. T. (2005). A featuresalience analogue of the inverse base-rate effect. Korean Journal of Thinking \& Problem Solving, 15, 17-28.

Erickson, M. A., \& KruschKe, J. K. (1998). Rules and exemplars in category learning. Journal of Experimental Psychology: General, 127, 107-140.

Fagot, J., Kruschke, J. K., Dépy, D., \& Vauclair, J. (1998). Associative learning in baboons (Papio papio) and humans (Homo sapiens): Species differences in learned attention to visual features. Animal Cognition, 1, 123-133.

GLUCK, M. A. (1992). Stimulus sampling and distributed representations in adaptive network theories of learning. In A. Healy, S. Kosslyn, \& R. Shiffrin (Eds.), Essays in honor of William K. Estes (Vol. 1, pp. 169-199). Hillsdale, NJ: Erlbaum.

GLUCK, M. A., \& Bower, G. H. (1988). From conditioning to category learning: An adaptive network model. Journal of Experimental Psychology: General, 117, 227-247.

Juslin, P., Wennerholm, P., \& Winman, A. (2001). High-level reasoning and base-rate use: Do we need cue-competition to explain the inverse base-rate effect? Journal of Experimental Psychology: Learning, Memory, \& Cognition, 27, 849-871.

KAHNEMAN, D., \& TVERSKY, A. (1973). On the psychology of prediction. Psychological Review, 80, 237-251.

Kalish, M. L. (2001). An inverse base rate effect with continuously valued stimuli. Memory \& Cognition, 29, 587-597.

KRUSCHKE, J. K. (1992). ALCOVE: An exemplar-based connectionist model of category learning. Psychological Review, 99, 22-44.

KRUSCHKE, J. K. (1996). Base rates in category learning. Journal of Experimental Psychology: Learning, Memory, \& Cognition, 22, 3-26.

KruschKe, J. K. (2001). The inverse base-rate effect is not explained by eliminative inference. Journal of Experimental Psychology: Learning, Memory, \& Cognition, 27, 1385-1400.

KRUSCHKE, J. K. (2003). Attentional theory is a viable explanation of the inverse base-rate effect: A reply to Winman, Wennerholm, and Juslin (2003). Journal of Experimental Psychology: Learning, Memory, \& Cognition, 29, 1396-1400.

Kruschie, J. K., Kappenman, E. S., \& Hetrick, W. P. (2005). Eye gaze and individual differences consistent with learned attention in associative blocking and highlighting. Journal of Experimental Psychology: Learning, Memory, \& Cognition, 31, 830-845.

Markman, A. B. (1989). LMS rules and the inverse base-rate effect: Comment on Gluck and Bower (1988). Journal of Experimental Psychology: General, 118, 417-421.

Medin, D. L., \& Bettger, J. G. (1991). Sensitivity to changes in baserate information. American Journal of Psychology, 104, 311-332.

Medin, D. L., \& Edelson, S. M. (1988). Problem structure and the use of base-rate information from experience. Journal of Experimental Psychology: General, 117, 68-85.

Medin, D. L., \& SchafFer, M. M. (1978). Context theory of classification. Psychological Review, 85, 207-238.

Myers, J. L., Lohmeier, J. H., \& Well, A. D. (1994). Modeling probabilistic categorization data: Exemplar memory and connectionist nets. Psychological Science, 5, 83-89.
Nelson, M. W. (1993). The effects of error frequency and accounting knowledge on error diagnosis in analytical review. Accounting Review, 68, 804-824

Nosofsky, R. M. (1984). Choice, similarity, and the context theory of classification. Journal of Experimental Psychology: Learning, Memory, \& Cognition, 10, 104-114.

Nosofsky, R. M. (1986). Attention, similarity, and the identificationcategorization relationship. Journal of Experimental Psychology: General, 115, 39-57.

Shanks, D. R. (1992). Connectionist accounts of the inverse base-rate effect in categorization. Connection Science, 4, 3-18.

Shanks, D. R., \& Darby, R. J. (1998). Feature- and rule-based generalization in human associative learning. Journal of Experimental Psychology: Animal Behavior Processes, 24, 405-415.

WiCKENS, T. D. (1989). Multiway contingency tables analysis for the social sciences. Hillsdale, NJ: Erlbaum.

Winman, A., Wennerholm, P., \& Juslin, P. (2003). Can attentional theory explain the inverse base-rate effect? Comment on Kruschke (2001). Journal of Experimental Psychology: Learning, Memory, \& Cognition, 29, 1390-1395.

Winman, A., Wennerholm, P., Juslin, P., \& Shanks, D. R. (2005). Evidence for rule-based processes in the inverse base-rate effect. Quarterly Journal of Experimental Psychology, 58A, 789-815.

\section{NOTES}

1. Pay was roughly proportional to participation time for the conditions in Experiments 1 and 2: decision-making $(£ 0)<$ memorization $(£ 1)<$ observational/feedback learning (£3).

2. The (C)ommon response proportion .35 was used to generate the expected values for $\chi^{2}$, which has two degrees of freedom because there were two different cases of $\mathrm{PC}+\mathrm{PR}$ and, hence, three possible response patterns to these two cases: CC, CR, and RR. A Monte Carlo statistical resampling procedure that was more formally appropriate indicated that these conclusions were conservative, that the actual $p$ values were near .0004 and .016 , respectively, and that the results for both conditions would still be at least marginally significant $(p=.006$ and .12 , respectively), even based on a more moderate IBRE of (C)ommon = .40 and $(\mathrm{R})$ are $=.60$

3. It was possible to assess base rate neglect directly here because the category structure (Table 3 ) included the disjoint-cues category structure as a subset (Figure 1, bottom) but also included the mirror opposite of that structure - that is, instances in which only one (P)erfectly predictive cue was assigned to a (C)ommon outcome but two different (P)erfectly predictive cues were assigned to a $(\mathrm{R})$ are outcome. $\mathrm{PC}_{0.5}+\mathrm{PR}_{1}$ testing trials used cues from within the disjoint-cues category substructure and provided strong evidence of the IBRE, whereas $\mathrm{PC}_{1}+\mathrm{PR}_{1}$ and $\mathrm{PC}_{0.5}+\mathrm{PR}_{0.5}$ combined cues from the two different substructures and provided strong evidence of base rate neglect.

4. All the participants were unpaid, since Experiment 3 was an attempt to find the simplest possible conditions for the IBRE. The summary of all the experiments and conditions in Table 7 shows that paid/ unpaid conditions did not covary with the presence/absence of the IBRE.

5. We actually had an additional 18 participants in this condition, who had also done a trial-by-trial learning version of the inverse base rate effect with perceptual stimuli, rather than symptoms and diseases. Since the learning experiment with perceptual stimuli resulted in a significant IBRE, it is, in some senses, more impressive that these participants had results that were qualitatively similar to those for the naive participantsthat is, no IBRE: $\mathrm{C}=.72$ for $\mathrm{I}, \mathrm{C}=.56$ for $\mathrm{PC}+\mathrm{PR}$, and $\mathrm{C}=.78$ for $\mathrm{I}+\mathrm{PC}+\mathrm{PR}$. In brief, the lack of an IBRE for these results is unlikely to have been a consequence of a lack of power. 


\section{APPENDIXA \\ Sample Instructions in Experiment 1}

The instructions for one of the versions of Experiment 1's pure decision-making condition:

"You are a medical doctor in training who has just seen a series of patients. All of the patients have had some combination of dizziness, sore muscles and stuffy nose and suffer from either terrigitis or coralgia. Note that these diseases are fictitious. In particular, you have noticed that patients with the combination of symptoms dizziness and stuffy nose have always had coralgia and patients with the combination of symptoms sore muscles and dizziness have always had terrigitis. All of your patients so far have had one of these two combinations of symptoms and diseases. Also you have noticed that approximately three times as many patients have had terrigitis as have had coralgia.

To summarize, approximately three times as many patients have exhibited the symptoms sore muscles and dizziness and had terrigitis as have exhibited the symptoms stuffy nose and dizziness and had coralgia.

Once you have read this carefully, please circle below what disease you think each new patient is likely to have given their symptoms. Please only choose a single disease for each patient:" [This was followed by the test cases.]

APPENDIX B

Observational-Learning Replication of the Standard Inverse Base Rate Effect Task

Table B1

Testing Trial Response Proportions

\begin{tabular}{|c|c|c|c|c|}
\hline \multirow[b]{2}{*}{ Symptoms } & \multicolumn{4}{|c|}{ Choice Proportions } \\
\hline & (C)ommon & (R)are & $\mathrm{Co}$ & Ro \\
\hline $\mathrm{I}+\mathrm{PC}$ & $.898(.980)$ & $.086(-)$ & $.000(-)$ & $.016(-)$ \\
\hline $\mathrm{I}+\mathrm{PR}$ & $.086(-)$ & $.867(.954)$ & $.023(-)$ & $.023(-)$ \\
\hline I & $.688(.746)$ & $.203(.174)$ & $.063(.049)$ & $.047(.031)$ \\
\hline $\mathrm{PC}$ & $.969(.933)$ & $.031(.031)$ & $.000(.031)$ & $.000(.004)$ \\
\hline PR & $.063(.040)$ & $.875(.911)$ & $.000(.018)$ & $.063(.031)$ \\
\hline $\mathbf{P C}+\mathbf{P R}$ & $.406(.353)$ & $.563(.612)$ & $.000(.022)$ & $.031(.013)$ \\
\hline $\mathrm{I}+\mathrm{PC}+\mathrm{PR}$ & $.516(.580)$ & $.453(.402)$ & $.016(.013)$ & $.016(.004)$ \\
\hline $\mathrm{I}+\mathrm{PCo}$ & $.313(.406)$ & $.109(.080)$ & $.531(.469)$ & $.047(.045)$ \\
\hline $\mathrm{I}+\mathrm{PRo}$ & $.158(.219)$ & $.127(.085)$ & $.000(.031)$ & $.716(.665)$ \\
\hline $\mathrm{PC}+\mathrm{PRo}$ & $.313(.353)$ & $.047(.027)$ & $.094(.058)$ & $.547(.563)$ \\
\hline $\mathrm{I}+\mathrm{PC}+\mathrm{PRo}$ & $.734(.719)$ & $.172(.036)$ & $.016(.036)$ & $.078(.210)$ \\
\hline $\mathrm{I}+\mathrm{PR}+\mathrm{PCo}$ & $.172(-)$ & $.656(-)$ & $.141(-)$ & $.031(-)$ \\
\hline $\mathrm{Io}+\mathrm{PC}+\mathrm{PR}$ & $.297(-)$ & $.516(-)$ & $.094(-)$ & $.094(-)$ \\
\hline $\mathrm{PC}+\mathrm{PR}+\mathrm{PCo}$ & $.219(-)$ & $.484(-)$ & $.250(-)$ & $.047(-)$ \\
\hline $\mathrm{PC}+\mathrm{PR}+\mathrm{PRo}$ & $.234(-)$ & $.391(-)$ & $.047(-)$ & $.328(-)$ \\
\hline $\mathrm{I}+\mathrm{Io}$ & $.188(-)$ & $.156(-)$ & $.469(-)$ & $.188(-)$ \\
\hline $\mathrm{I}+\mathrm{PC}+\mathrm{Io}$ & $.828(-)$ & $.047(-)$ & $.016(-)$ & $.109(-)$ \\
\hline $\mathrm{I}+\mathrm{PR}+\mathrm{Io}$ & $.096(-)$ & $.746(-)$ & $.063(-)$ & $.095(-)$ \\
\hline $\mathrm{PC}+\mathrm{PCo}$ & $.531(-)$ & $.031(-)$ & $.406(-)$ & $.031(-)$ \\
\hline $\mathrm{PR}+\mathrm{PRo}$ & $.031(-)$ & $.469(-)$ & $.063(-)$ & $.438(-)$ \\
\hline $\mathrm{I}+\mathrm{PC}+\mathrm{PCo}$ & $.719(-)$ & $.063(-)$ & $.156(-)$ & $.063(-)$ \\
\hline $\mathrm{I}+\mathrm{PR}+\mathrm{PRo}$ & $.063(-)$ & $.656(-)$ & $.063(-)$ & $.219(-)$ \\
\hline No cues & $.323(-)$ & $.194(-)$ & $.323(-)$ & $.161(-)$ \\
\hline
\end{tabular}

Note- "PC" refers to a (P)erfectly predictive cue of a (C)ommon outcome, "PR" to a (P)erfectly predictive cue of a (R)are outcome, and "I" to an (I)mperfectly predictive/shared cue. "Co" and "Ro" refer to other (C)ommon and (R)are responses that were possible. The numbers in parentheses are from Kruschke (1996, Experiment 1), a standard replication of the inverse base rate effect.

(Manuscript received June 23, 2006; revision accepted for publication September 15, 2006.) 